EPJ Web of Conferences 64, 09001 (2014)

DOI: $10.1051 /$ epjconf/ 20146409001

(C) Owned by the authors, published by EDP Sciences, 2014

\title{
The magnetospheric accretion/ejection process in young stellar objects: open issues and perspectives
}

\author{
J. Bouvier ${ }^{1, a}$ \\ ${ }^{1}$ UJF-Grenoble 1 / CNRS-INSU, Institut de Planétologie et d'Astrophysique de Grenoble (IPAG) UMR 5274, \\ Grenoble, F-38041, France
}

\begin{abstract}
This summary talk aims at highlighting some of the remaining open issues regarding the physics of the magnetospheric accretion/ejection process in young stellar objects (YSOs). It lists a number of questions related to YSOs magnetic fields and accretion regimes, the structure and properties of accretion shocks, the origin of multiple outflow components, and the impact of the star-disk magnetic interaction onto early angular momentum evolution.
\end{abstract}

\section{Introduction}

The presentations at this conference have clearly illustrated the new insights theory, numerical simulations, and observations have brought to our understanding of the magnetic star-disk interaction process in young stellar objects (YSOs). I shall rely on these presentations to outline in this summary talk some of the remaining issues to be addressed in the forthcoming years regarding the origin and properties of YSOs magnetic fields, the eventuality of different accretion regimes, the physics of accretion shocks, the coexistence of several outflow components, and their impact on angular momentum evolution.

\section{YSOs magnetic fields}

While significant progress has been made on the derivation of magnetic field strength and topology in YSOs ( cf. contributions to this volume by S. Gregory, C. Johns-Krull, and S. Hubrig), many aspects remain to be addressed, including the following ones:

- What kind of dynamos operates in fully convective and partly radiative young stars?

- How does disk accretion impact on the large scale magnetic field topology?

- What is the short term evolution of magnetic fields (e.g., magnetic cycles, polarity reversals, etc.)? How does it impact on accretion flows?

- How to relate magnetic field properties to stellar parameters (mass, age, rotation, Macc, etc.)

- What is the relative importance of large scale vs. compact magnetic field components?

\footnotetext{
ae-mail: Jerome.Bouvier@obs.ujf-grenoble.fr
} 
- Do accreting and non-accreting young stars share the same magnetic field properties?

On the observational side, large-scale campaigns aiming at measuring the magnetic field strength and topology of YSOs over a wide mass range and covering various evolutionary stages, from embedded protostars to Class III sources, are required to settle these issues. On the theoretical side, numerical simulations of fully convective dynamos in rapidly rotating stars, possibly integrating the interaction with a circumstellar disk as an outer boundary condition, would provide clues to the magnetic field generation in young stars.

\section{Accretion regimes}

The last 20 years have seen a shift in paradigm from boundary layer accretion to magnetospheric accretion. Recent observational and numerical studies, however, tend to suggest that different accretion regimes may operate in young stars (cf. contributions to this volume by A.-M. Cody, S. Alencar, N. Fonseca, R. Kurosawa, R. Lovelace, and M. Romanova). This opens the intriguing possibility that a young star may experience different accretion phases during its early pre-main sequence evolution, let alone the rare but intense FU Ori events. In turn, this new perspective rises a number of issues:

- Through how many different ways can accretion occur onto the stellar surface: stable, unstable, propeller, quiescent?

- How to relate the different accretion regimes to the system's properties (accretion rate, magnetic field strength, topology, and obliquity, mass, rotation rate, etc.)?

- What is the observational evidence for different accretion regimes? Can different accretion regimes coexist in the same system?

- Is accretion steady-state or episodic? On which timescales?

Long-term spectrophotometric monitoring of ensembles of YSOs is essential to better understand the different accretion modes in these systems. Also, identifying a few prototypical systems exhibiting (or alternating between) the various accretion regimes would be of great interest. Obviously, the continuing development of 3D numerical simulations of the magnetic star-disk interaction will provide strong insight into these issues, as they explore different mass accretion rates and magnetic topologies, and include evermore refined physics.

\section{Accretion shocks}

Renewed accretion shock models have been flourishing over the last years, mostly through the development of 2D numerical simulations of accretion funnel flows reaching the stellar surface at free fall velocity (cf. contributions to this volume by L. de Sà and T. Matsakos). In parallel, X-ray observations of YSOs have recently provided a new and powerful diagnostics of the physics of accretion shocks (cf. R. Bonito's contribution to this volume). Current developments will help addressing the following issues:

- What is the structure, stability, and evolution of accretion shocks at the surface of YSOs?

- What observational diagnostics are best suited to investigate their physics? What can we learn from $\mathrm{X}$-ray spectra and UV line profiles?

- What is the relative importance of compact vs. large-scale magnetic components in governing the accretion shock structure?

- Do different accretion regimes translate into different shock properties? 
- Where does the energy dissipated in the accretion shock go (stellar interior, outflows, radiation, etc.)? How does it impact early stellar evolution and disk chemistry/evolution?

The on-going effort to combine MHD numerical simulations with radiative transfer computations is sorely needed to be able to provide quantitative predictions of observable diagnostics, such as continuum excess flux, emission line flux and profiles, as well as their expected temporal variability. These predictions will prove extremely useful to design observations that will efficiently explore the physics of accretion shocks.

\section{Outflows}

Outflows are intimately related to the accretion process in YSOs. The tight relationship observed between the strength of accretion and outflow diagnostics clearly indicates that accretion power drives the ejection process(es). Beyond this general statement, many outflow models have been proposed (cf. contributions to this volume by P. Lii, M. Romanova, C. Zanni, J. Staff, and B. Stelzer) and major issues remain indeed regarding the identification of the different outflow components and how each of them relates to the accretion process:

- How many outflow components are present in an accreting YSO system? Where do they originate from?

- How can we distinguish (observationally) between accretion driven stellar winds, interface outflows (e.g. magnetospheric ejections, X-winds), disk winds?

- How is each component powered (by accretion)? How does each component contribute to the angular momentum budget of the system?

- Are outflows episodic or steady-state? On which timescales?

YSOs offer a number of outflow diagnostics (e.g. blueshifted absorptions in emission line profiles, forbidden emission lines, ionized jets, etc.) and the radiative transfer post-processing of MHD outflow simulations starts to yield specific predictions for these diagnostics. The exploration of the time domain appears today as one of the most promising directions to improve our understanding of the accretion/ejection process in YSOs. By monitoring simultaneously accretion and ejection diagnostics, one may hope to directly relate outflow events to accretion bursts, and link their characteristic variation timescales to spatial scales, thus providing clues to the regions where they originate.

\section{Angular momentum evolution}

One of the greatest challenges in star formation and early stellar evolution is to understand the low angular momentum content of newly-born stars. The observational evidence that accreting stars have on average lower spin rates that non accreting ones suggests that the magnetic star-disk interaction process acts to brake the central object (see R. Pudritz's and F. Gallet's contributions to this volume). Yet, the physical mechanisms(s) by which enough angular momentum can be removed from the young star in order to prevent it from spinning up as it accretes material from its circumstellar disk is far from being understood. Among the most pressing issues, one may list:

- What is the net (time averaged) torque exerted on the central star by the magnetically-driven accretion/ejection process? Can it be negative for a few million years, as observations suggest? What are the dominant spin-down processes?

- Is the spin evolution of pre-main sequence stars monotonic, or are there long-term spin-up/spindown episodes? 
- How reliable is the evidence for an accretion-rotation connection among young stars? How does it depend on stellar parameters (mass, accretion rate, magnetic fields, etc.)?

- What does the instantaneous rotation rate of a young star tell us about its accretion history? What is the impact of different accretion regimes on the stellar spin?

Long-term 3D MHD simulations of the star-disk interaction are needed to predict the angular momentum exchange associated to the accretion/ejection process and the fate of the stellar spin. Observationally, a clear understanding of the accretion-rotation connection awaits more precise measurements of mass accretion rates and magnetic field properties, as well as the determination of the gaseous disk's inner radius relative to the corotation radius, either directly through long baseline interferometry or indirectly through the analysis of spectral features formed at the disk inner edge.

\section{Conclusion}

The magnetic star-disk interaction process is at the heart of YSOs physics. Indeed, it accounts for most of their observed properties. Understanding the details of this complex, magnetically-mediated interplay between the central star and its surrounding disk and outflows is therefore one of the ultimate goals in star formation research. The time is ripe for a detailed confrontation between in-depth modeling (e.g., time-dependent 3D MHD simulations coupled to radiative transfer models) and observations (e.g., intense spectrophotometric monitoring of accretion and ejection diagnostics of selected YSOs). This offers a promising direction to unveil the structure and physics of the inner 0.1 AU region of young stellar systems. Within a few years timescale, the magnetic properties of YSOs will eventually be fully documented, thanks to a combination of observational techniques including high-resolution spectroscopy and spectropolarimetry in the optical and the infrared, while the inner disk structure will be further constrained from long baseline spectro-interferometry. The parallel advances of observations and models thus offer the best promise for rapid progress in our understanding of the magnetic star-disk interaction process in young stellar systems.

\section{Acknowledgements}

It is a pleasure to thank the organisers for a fruitful and enjoyable conference. This contribution was supported by grant ANR 2011 Blanc SIMI5-6 02001 "Toupies: Towards understanding the spin evolution of stars" (http: //ipag.osug.fr/Anr_Toupies/). 